\title{
Application of High-Speed Wind Tunnel Control Based on PID Neural Network
}

\author{
Li Ji ${ }^{1, a}$, Hong Wang ${ }^{2, b}$ and Hailong Huang ${ }^{3, c}$ \\ ${ }^{1,2,3}$ School of Mechanical Engineering \& Automation, Northeastern University, Shenyang 110004, \\ China \\ a jilirory0211@gmail.com, ${ }^{b}$ hongwang@mail.neu.edu.cn, ${ }^{c}$ huanghailonghhl@126.com
}

Keywords: PID neural network, High-Speed wind tunnel, Mach number

\begin{abstract}
The traditional PID control in nonlinear system such as high-speed wind tunnel has limitations, and the range of using is limited. The BP neural network has been widely applied to the optimization of the PID controller parameter adjustment. The PID neural network control system is introduced in the conventional PID control, which has advantages such as simple structure, physical meaning clear parameters, but also has a neural network of parallel structure and the function of learning and memory and nonlinear mapping capability. The controller uses BP (error back propagation) algorithm to correct connection weights, through on-line training and learning and make objective function to achieve optimal value. This improvement scheme can not only improve algorithm in the training process, and the convergence speed in the wind tunnel, the control valve control system response speed, high precision, meet the steady-state real-time control requirements.
\end{abstract}

\section{Introduction}

The PID controller is the earliest development of classical control theory and application of the control strategy. Because one of the methods is simple and good robustness and high reliability. It is widely used in industrial process and obtained good control effect. With the development of industry, the object of complex deepening, especially for the delay, time-varying, nonlinear of complex system, the traditional PID control seems powerless. So the application of traditional PID control has been limited and challenges. The application of neural network control system improved the system of information system processing power and ability to adapt and the intelligent level of system. In addition, the neural network has an ability of approximated any continuous bounded nonlinear function, to nonlinear system and uncertainty system [2]. It is a kind of new effective ways to solve the problem. PID control composed of the deviation of proportional (P) integral (I) and derivative (D) a linear combination of the control measure, and the object is controlled. Therefore, in order to get better control effect from PID controller, you will have to adjust good proportional, integral and derivative three control function, form control to coordinate with each other in mutual restrict relationship. But, the relationship is not always a simple linear combination. The neural network based on neurons has any nonlinear approximation ability. We can use neural network by the performance of the system to achieve optimal combination with learning the PID control.

\section{The Model of PID Neural Network}

The structure of BP network is shown in figure 1 below. The figure shows that the BP network is one kind that has the three layers, including input neural network layer, layer (hidden layer) and output layer. The up and down layer achieve all connection, but each layer between neurons without connection [1]. 


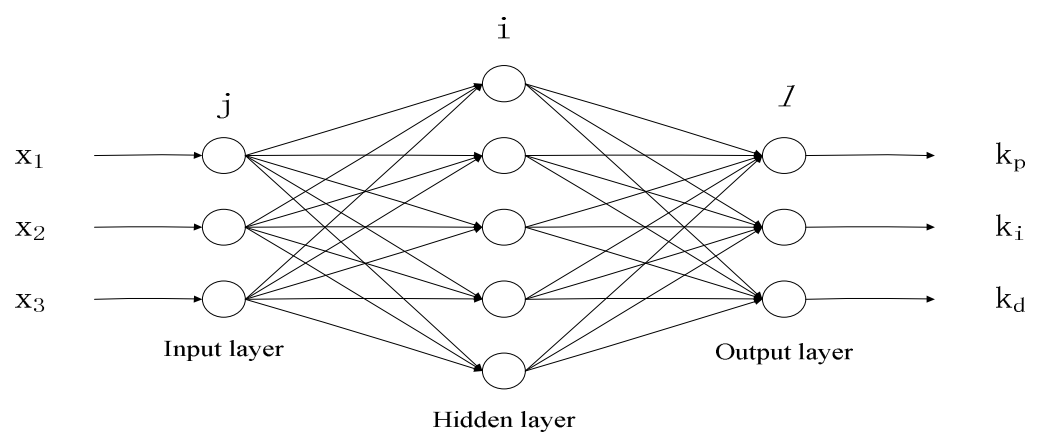

Fig. 1. Neural network structure

When a pair of learning samples available to the network, the activation of neurons transfers from the input layer by the interface layer to the output layer. According to reducing the error between output and the actual output direction, which from the output layer after layer back to reverse coating to input the weights between corrections step by step, this algorithm called "error back propagation method", that is the BP algorithm. With the spread of the error correction unceasingly to reverse, the network to the input mode ring accuracy is rising. And the error back propagation of transfer function in the derivation of BP network project transfer function is different from perceptions model. So we can't use the perception of the network hard threshold transfer function, and commonly have sigmoid type logarithm, tangent function or linear function. Due to differential of the transfer function, so, on the one hand, the division area of the BP network is no longer a linear points, but composite area of a nonlinear hyper plane. On the other hand, the network can strictly adopted descend method for the study, weights fixed analytical formula is very clear[5]. Neurons are the creatures of neurons a simulation and simplified. It is the basic processing unit neural network.

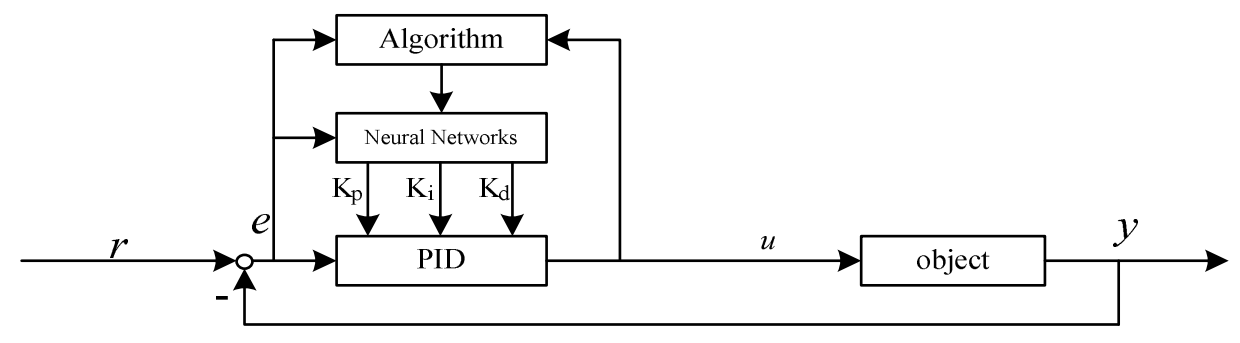

Fig. 2. Architecture of PID control system based on BP neural network

We set large delay and the nonlinear slowly-varying, wind tunnel system as the controlled object, with Matlab simulation experiment to test neural network PID control system performance. We have a architecture of PID control system based on BP neural network as shown in the Fig.2.

\section{The Application of the BP Neural Network PID Control in Wind Tunnel the Mach Number}

The wind tunnel test section size is $1.6 \mathrm{~m}$ x $1.5 \mathrm{~m}$, the design of testing Mach number range of 0.3 $\sim 1.2$, and $1.35 \sim 2.25$. Subsonic Mach number $(0.3 \sim 0.8)$ can not only is controlled by the second larynx way, and also controlled through the governing valve of the adjusting of sound velocity, cross Mach number $(1.0 \sim 1.2)$ is controlled through the flap of the main flow of in room gas ejector realizing perfect, Mach number $(1.35 \sim 2.25)$ is changed through the different solid piece of nozzle realized. The highest pressure of the front room is $0.4 \mathrm{Mpa}$, the Mach number is 0.8 , The Reynolds number of regular speed under pressure is $2.6 \times 106$ (reference length is one meters). The wind tunnel profile structure shown in the Fig.3. 


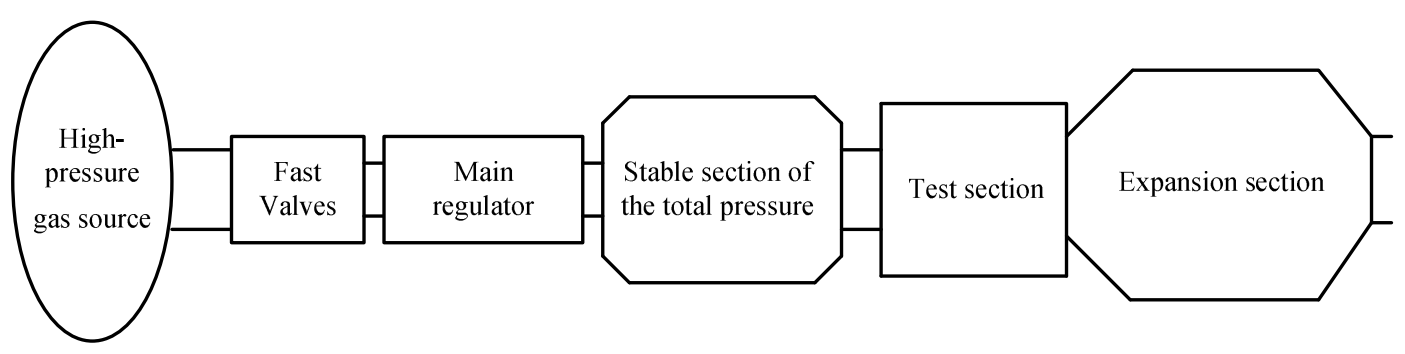

Fig. 3. Wind tunnel profile structure

The Transfer Function. The control object transfer function of wind tunnel across the supersonic can generally indicate:

$$
\frac{Y(s)}{u(s)}=\frac{K e^{-\tau s}}{T s+1}
$$

or indicate:

$$
\frac{Y(s)}{u(s)}=\frac{K e^{-\tau s}}{\left(T_{1} s+1\right)\left(T_{2}+1\right)}
$$

In the formula, $\mathrm{Y}(\mathrm{s})$-the output of the control objects, is generally the Mach number or stable period of total pressure. $U$ (s)-control parameters;

T,T1 and T2-the control object, the inherent time constant, generally is different from the running state of wind tunnel. $\tau$-pure lag time, generally for $0.1 \sim 0.6 \mathrm{~s}$. K- The open-loop transfer functions of system [4].

Matlab Simulation Curves.In order to obtain a visual interface, debugging program comfortably, to achieve the optimal form interface and easy to demonstrate, We use the Matlab GUI functions designed to provide the following presentation interface(Abscissa is time, in seconds, vertical axis is the Mach number)[3].

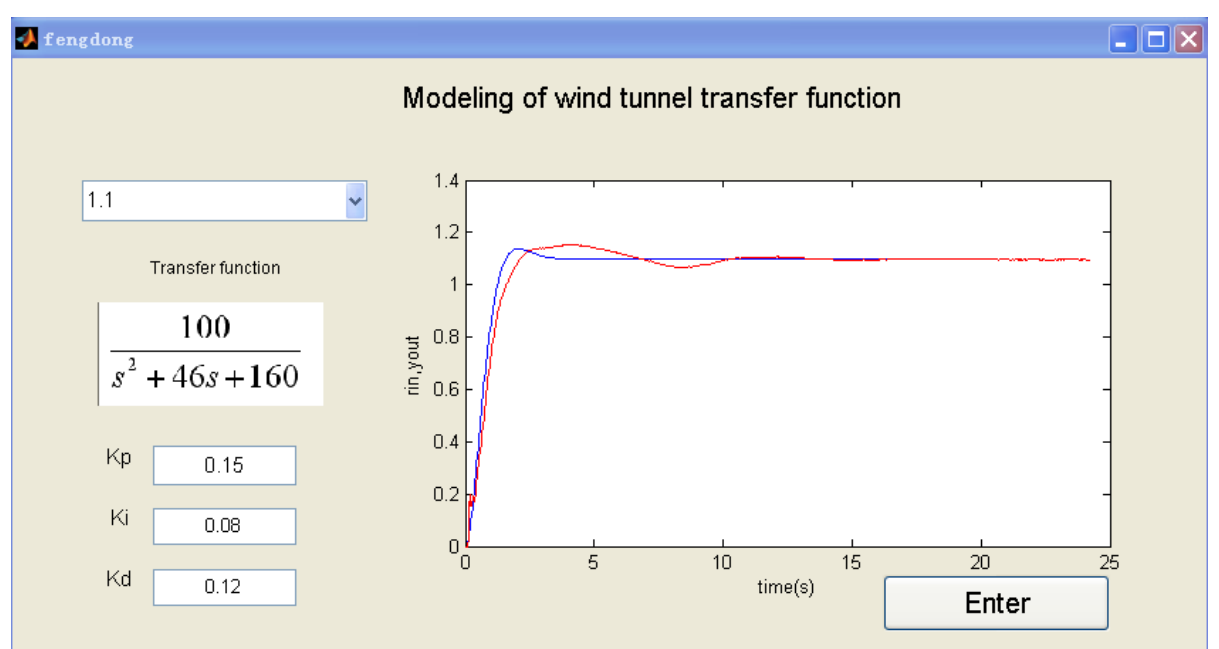

Fig. 4. Experiment simulation results of PID and PIDNN control system in 1.1 Mach number 


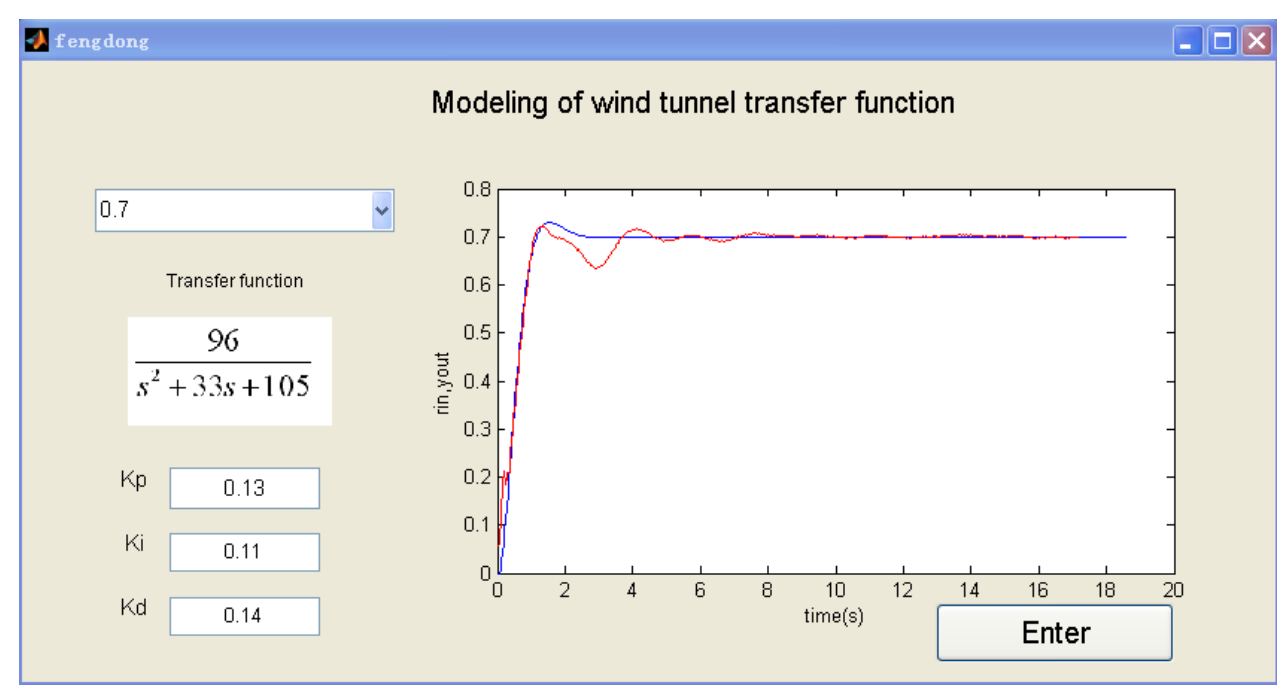

Fig. 5. Experiment simulation results of PID and PIDNN control system in 0.7 Mach number

The figure 4 is the simulation curves under the Mach 1.1, and the figure 5 is the simulation curves under the mach 0.7. Red curve is the traditional PID control curve; the blue curve is the use of neural network and PID combination to get the curve [6]. Neural network PID control than the conventional PID control and BP neural network PID control compared to the response speed is significantly faster than the other two control methods, the overshoot and settling time is also a great advantage.

\section{Conclusion}

Through the above analysis and simulation, the neural network PID control compared with the traditional PID control. It is concluded from the simulation experiments that the neural network PID control almost do not produce overshoot, the whole transition process more smoothly, and the transition time is better than the traditional PID control time. The BP neural network and the PID control, the way of the combination of the will need a lot of data, can we reach the ideal training transfer function. The testing Mach number convergence rapidly, in the $5 \mathrm{~s}$ can reach stable the Mach number. Neural network PID has been used in wind tunnel control system that has good control effect.

\section{References}

[1] J.K.Liu:Matlab simulation of advanced PID control,second ed.Publishing House of Electronics Industry,China, 2004.

[2] Y.H.Cao,S.C.Li and Y.C.Liu:Control Engineering of China.Vol.14.(2007),pp.38-40.

[3] Y.J.Shen,X.S.Gu: Journal of East China University of Science and Technology(Natural Science Edition).Vol.32.No.7(2006),pp.860-863.

[4] Y.Fu:Nonlinear Adaptive Decouping Control Based on Multiple Models and Neural Networks.

[5] A.R.Kabiri-Samania,J.Aghaee-Tarazjani,S.M,Borghei,D.S.Jeng:Applied Soft Computing. 11 (2011),pp.2880-2887.

[6] Y.G.Liu, B.B.Liu,S.H.Ling: Neurocomputing.74(2011),pp.1062-1068. 